\title{
Dynamic mitochondrial changes during differentiation of P19 embryonic carcinoma cells into cardiomyocytes
}

\author{
JIN JIN $^{1 *}$, QIN-KAO XUAN ${ }^{1 *}$, LI-JUAN ZHOU ${ }^{1}$, CHUN-MEI SHI $^{2}$, \\ GUI-XIAN SONG ${ }^{1}$, YAN-HUI SHENG ${ }^{1}$ and LING-MEI QIAN ${ }^{1}$ \\ ${ }^{1}$ Department of Cardiology, The First Affiliated Hospital of Nanjing Medical University; \\ ${ }^{2}$ State Key Laboratory of Reproductive Medicine, Department of Pediatrics, Nanjing Maternity and \\ Child Health Hospital Affiliated to Nanjing Medical University, Nanjing 210029, P.R. China
}

Received December 12, 2013; Accepted May 2, 2014

DOI: $10.3892 / \mathrm{mmr} .2014 .2315$

\begin{abstract}
Murine P19 embryonal carcinoma cells are multipotent cells that can differentiate into cardiomyocytes when treated with dimethyl sulfoxide. This experimental model provides an invaluable tool to study different aspects of cardiac differentiation, such as the function of cardiac-specific transcription factors and signaling pathways, and the regulation of contractile protein expression. The role of mitochondria during cardiac differentiation is unclear. In this context, we have examined the mitochondrial-related changes in undifferentiated and differentiated P19 cells. We observed that mitochondrial DNA content sharply decreased in P19 cell aggregates compared to undifferentiated cells, accompanied by decreased levels of adenosine triphosphate (ATP) and reactive oxygen species (ROS). Following the aggregation stage, the mitochondrial DNA content reached its highest level on day 7 of the differentiation process, with the intracellular ROS level showing a trend to increase, similar to cellular ATP production. In conclusion, our study on differentiating P19 embryonal carcinoma cells provides new insights into the role of mitochondria in the differentiation of P19 stem cells into beating cardiomyocytes.
\end{abstract}

\section{Introduction}

Heart diseases, including coronary heart disease, heart failure and cardiomyopathy, have become leading causes of death in the developed world (1-3). Important damage of the

Correspondence to: Professor Ling-Mei Qian or Professor Yan-Hui Sheng, Department of Cardiology, The First Affiliated Hospital of Nanjing Medical University, Guangzhou Road 300, Nanjing 210029, P.R. China

E-mail: 1mqian@njmu.edu.cn

E-mail: yhsheng@njmu.edu.cn

*Contributed equally

Key words: cardiomyocyte, mitochondria, P19 embryonal carcinoma cell myocardium is considered irreversible, since mature cardiomyocytes are highly differentiated and have limited regenerative capacity (4). In order to address this issue, cell regeneration therapy has emerged as a promising new approach for myocardial repair $(5,6)$. In this direction, a large body of studies have focused on understanding the mechanisms of cardiomyocyte differentiation using somatic and embryonic stem (ES) cells, as well as embryonic carcinoma cells (7-9). The P19 embryonal carcinoma cell line was derived from a teratocarcinoma induced in $\mathrm{C} 3 \mathrm{H} / \mathrm{HC}$ mice, and has been widely used as a model system to study the molecular mechanisms underlying cellular differentiation $(8,10)$. Although the P19 cell line has been extensively used to elucidate the mechanisms governing differentiation of stem cells into cardiomyocytes $(7,8,11)$, the role of mitochondria during this process has not been studied.

Mitochondria are membrane-bound organelles that possess their own genome and provide the majority of adenosine triphosphate (ATP) within eukaryotic cells $(12,13)$. The maternally-inherited, $16.6-\mathrm{kb}$ mitochondrial genome encodes 13 subunits of the electron-transfer chain, 22 transfer ( $t$ )RNAs and two ribosomal (r)RNAs $(13,14)$. Mitochondria are involved in numerous processes central to cellular functions, including calcium signaling, growth and differentiation, cell-cycle control and cell death (15). Previous reports have suggested a role of mitochondria in ES cell viability and differentiation, a finding that warrants further investigation (16-18).

This study used P19 embryonal carcinoma cells based on their ability to differentiate into cardiomyocytes in suspension culture when exposed to $1 \%$ dimethyl sulfoxide(DMSO) $(10,19)$. We compared the mitochondrial morphology and localization between undifferentiated and differentiated P19 cells, and quantified the mitochondrial DNA (mtDNA) copy number, ATP content and reactive oxygen species (ROS) production during cardiac differentiation. Our data provide evidence that mitochondria play an important role in the differentiation of cardiomyocytes.

\section{Materials and methods}

Cell cultures and differentiation. The P19 cells used in this study were obtained from the American Type Culture Collection (ATCC; Manassas, VA, USA), and were cultured 
in Gibco ${ }^{\circledR} \alpha$-modified Eagle's medium ( $\alpha$-MEM), supplemented with $10 \%$ Gibco $^{\circledR}$ fetal bovine serum (FBS) (both from Thermo Fisher Scientific Inc., Grand Island, NY, USA) and $1 \%$ penicillin/streptomycin, at $37^{\circ} \mathrm{C}$ in a $5 \% \mathrm{CO}_{2}$ atmosphere. In order to induce cardiac differentiation, P19 cells that had reached the exponential growth phase were trypsinized and transferred to $10-\mathrm{cm}$ bacterial dishes containing $\alpha$-MEM medium supplemented with $10 \% \mathrm{FBS}$ and $1 \%$ DMSO (Sigma-Aldrich, St. Louis, MO, USA) at a density of $10^{5}$ cells $/ \mathrm{m}$. The cells were left to aggregate for 4 days. The cell aggregates were then transferred into cell culture flasks containing complete medium containing 90\% $\alpha$-MEM, $10 \%$ FBS and $1 \%$ penicillin/streptomycin without DMSO. for an additional 7 days. The morphological changes in the P19 cells were examined and photographed under an inverted microscope (Nikon, Tokyo, Japan).

Western blotting. Total protein extracts were isolated from cultured cells, separated on a $10 \%$ sodium dodecyl sulfate gel by polyacrylamide gel electrophoresis, transferred onto a nitrocellulose membrane, and blocked in 5\% non-fat dried milk in a phosphate-buffered saline (PBS)/Tween-20 buffer. The membrane was then incubated with a polyclonal rabbit anti-cTnT antibody (1:500; Abcam, Cambridge, UK), and a polyclonal rabbit anti-tubulin antibody $(1: 10,000$; ABR-Affinity BioReagents cat no. AF0524; Affinity, Shanghai, China), followed by incubation with the secondary goat anti-rabbit $\mathrm{IgG}$ conjugated with horseradish peroxidase (1:10,000; ZSGB-Bio, Beijing, China).

Electron microscopy. Cultured cells were collected after trypsin (Gibco-BRL, Carlsbad, CA, USA) digestion, washed in fresh PBS (Wisent Inc., QC, Canada; $\mathrm{pH}$ 7.4), and fixed in a $2.5 \%$ glutaraldehyde- $4 \%$ paraformaldehyde solution. Cells were then washed in $0.1 \mathrm{M}$ cacodylate buffer, and post-fixed with $1 \%$ osmium tetroxide and $1.5 \%$ potassium ferrocyanide for $1 \mathrm{~h}$. Next, they were washed in water, stained with $1 \%$ aqueous uranyl acetate for $30 \mathrm{~min}$, and dehydrated through a graded series of ethanol (5 min in $70 \%$, $5 \mathrm{~min}$ in $90 \%$, and $5 \mathrm{~min}$ in 100\%). Finally, the samples were infiltrated and embedded in TAAB epon (Marivac Canada Inc., St. Laurent, Quebec, Canada). Ultrathin sections (60 $\mathrm{nm}$ ) were obtained on a Reichert Ultracut S microtome (Leica, Wetzlar, Germany), placed onto copper grids, stained with uranyl acetate and lead citrate, and examined under a transmission electron microscope (JEM-1010; JEOL, Tokyo, Japan), at an accelerating voltage of $80 \mathrm{kV}$.

Measurement of mtDNA concentration by quantitative (q) $P C R$. Relative levels of mtDNA were determined using qPCR, performed on an Applied Biosystems 7500 sequence detection system, and were quantified using the SDS software (both from Applied Biosystems, Foster City, CA, USA) in accordance with the manufacturer's protocol (20). Briefly, DNA was isolated from cells using a DNA extraction kit (cat no. A1120, Promega Corp., Madison, WI, USA) and quantified on a NanoDrop 2.0 spectrophotometer (Thermo Fisher Scientific Inc.). A 110-nucleotide mtDNA fragment within the cytochrome B gene $(C Y T B)$ was amplified with the following forward primer, reverse primer, and probe, respec- tively: 5'-TTT TAT CTG CAT CTG AGT TTA ATC CTG T-3', 5'-CCA CTT CAT CTT ACC ATT TAT TAT CGC-3', and AGC AAT CGT TCA CCT CCT CTT CCT CCA C. The sequences of the forward, reverse primer and probe for the amplification of a 291-bp region of the nuclear gene encoding the 28S rRNA, used for normalization, were respectively: 5'-GGC GGC CAA GCG TTC ATA G-3', 5'-AGG CGT TCA GTC ATA ATC CCA CAG-3', and TGG TAG CTT CGC CCC ATT GGC TCC T. The PCR product of $C Y T B$ had been previously cloned into the plasmid pMD 18-T (Takara Bio, Inc., Dalian, China) by qPCR and verified by DNA sequencing (Invitrogen, Shanghai, China). Plasmid standards (ND1-plasmid pMD 18-T; Takara Bio, Inc.) of known copy number for ND1 were used to generate a log-linear standard curve, from which the $C Y T B$ copy number was extrapolated. A standard curve from qPCR results on the plasmids that contained the $28 \mathrm{~S}$ fragment $\left(28 \mathrm{~S}-\mathrm{pMD} 18^{\circledR}-\mathrm{T}\right.$; Takara Bio, Inc.) was used to determine the copy number of the studied $28 S$ rRNA fragment. The ratio of mtDNA to nuclear DNA copies was used to define the concentration of mitochondria per cell.

Assessment of cellular ATP production. ATP was measured by a luciferase-based luminescence assay kit (Beyotime Institute of Biotechnology, Nantong, China). Briefly, cultured cells were homogenized in an ice-cold ATP-releasing buffer (Beyotime Institute of Biotechnology, cat no. S0026-4) and centrifuged at $12,000 \mathrm{x} \mathrm{g}$ for $10 \mathrm{~min}$. Following centrifugation, the supernatant was transferred to a fresh tube in order to measure the ATP concentration. A 20- $\mu 1$ sample along with $100 \mu \mathrm{l}$ of ATP detection buffer (Beyotime Institute of Biotechnology, cat no. S0026-1) were assayed in a single-tube luminometer (Turner BioSystems, Sunnyvale, CA, USA). The standard curve used to determine the ATP concentration $\left(10^{-3}-1 \mu \mathrm{M}\right)$ was prepared from a known amount of ATP, purchased from Beyotime Institute of Biotechnology. The relative ATP level was then normalized to the protein concentration of the respective sample, measured by the bicinchoninic acid assay method.

ROS assay. Intracellular ROS generation was measured using a 2',7'-dichlorodihydrofluorescein diacetate acetyl ester $\left(\mathrm{H}_{2}\right.$-DCFDA) probe (Sigma-Aldrich), as previously described (21). Briefly, cells were washed twice with PBS buffer and incubated at $37^{\circ} \mathrm{C}$ in prewarmed $\alpha$-MEM medium containing $5 \mu \mathrm{M} \mathrm{H}_{2}$-DCFDA. After $30 \mathrm{~min}$, the cells were washed three times with PBS. For flow cytometry, the cells were trypsinized and centrifuged at $500 \mathrm{x}$ g at $4^{\circ} \mathrm{C}$ for $5 \mathrm{~min}$, and resuspended in $300 \mu \mathrm{l}$ PBS. The cells were then analyzed by fluorescence-activated cell sorting (FACS), using a FACScan flow cytometer and the CellQuest software (both from BD Biosciences, San Jose, CA, USA).

Statistical analysis. Statistical significance of differences among experimental samples was assessed by a one-way analysis of variance (ANOVA), using the SPSS 16.0 statistical software (IBM, Armonk, NY, USA). All data were expressed as means \pm SD from three independent experiments. $\mathrm{P}<0.05$ was considered to indicate statistically significant differences. 
A
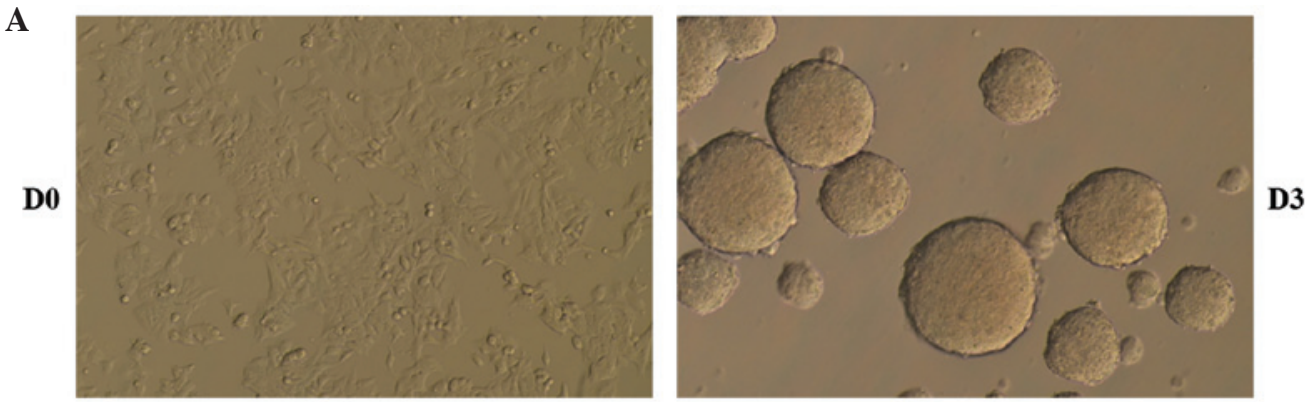

D3
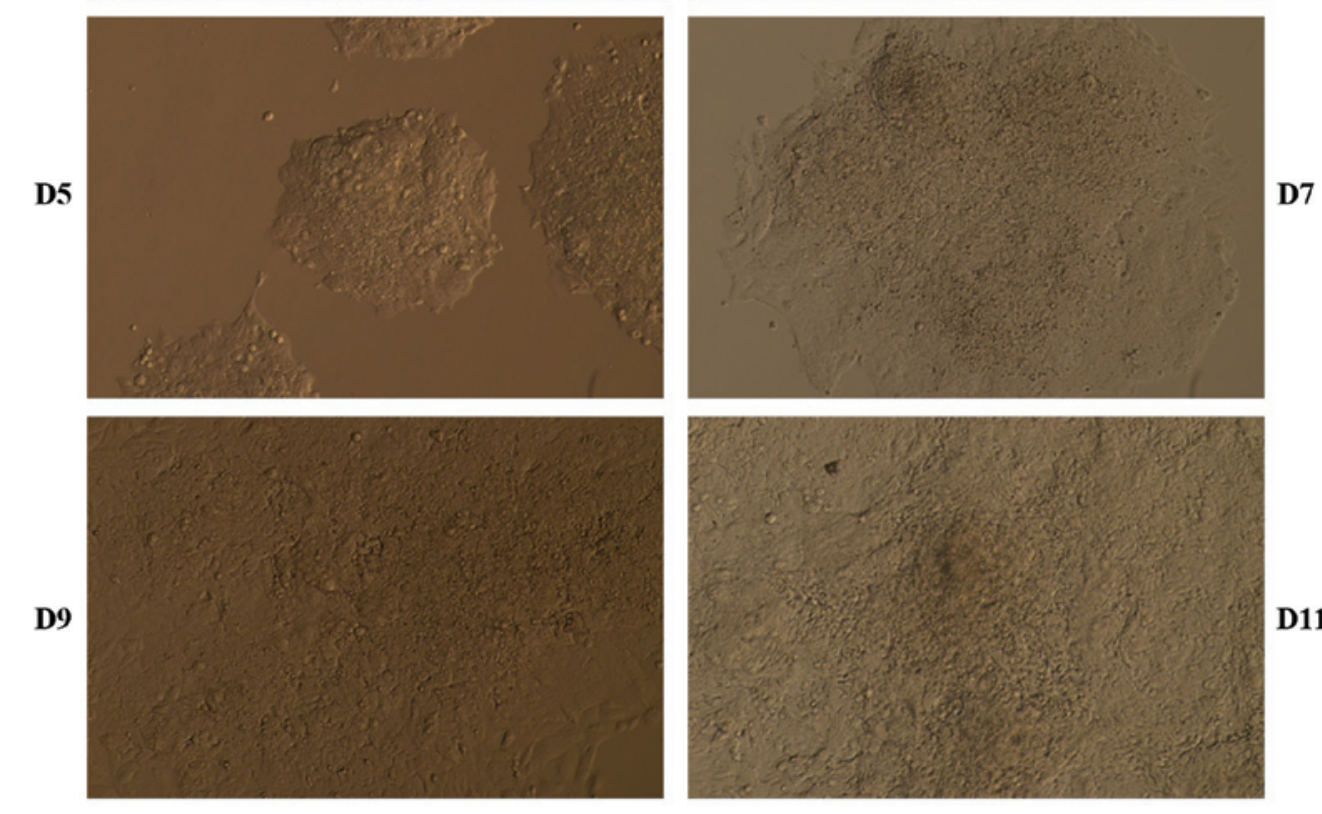

B

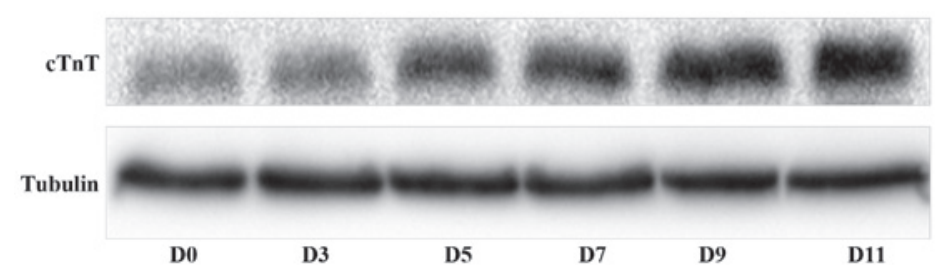

Figure 1. P19 embryonal carcinoma cell differentiation into cardiomyocytes on day (D)0, D3, D5, D7, D9, and D11. (A) Representative images of differentiating P19 cells. (B) In order to confirm the differentiation of P19 cells into mature cardiomyocytes, we used western blotting to quantify the expression level of a cardiomyocyte marker, the cardiac-specific isoform of troponin $\mathrm{T}(\mathrm{cTnT})$.

\section{Results}

Cardiac differentiation of P19 cells. Upon induction with $1 \%$ DMSO, cell aggregation, and a total of 11 days in culture, P19 cells differentiated into beating cardiomyocytes (Fig. 1A). When the cells were observed under a phase contrast microscope, differentiated cells displayed a denser cytoplasm, while the undifferentiated cells were relatively small. The first beating cardiomyocytes were observed on day 9 and were abundant by day 11 . In order to confirm the differentiation of P19 cells into mature cardiomyocytes, we analyzed the expression of the cardiomyocyte protein marker cTnT, encoding the cardiac-specific isoform of troponin T. Western blot analysis showed that the protein level of cTnT increased throughout cell differentiation (Fig. 1B).

Mitochondrial morphology and localization. The morphology and cellular localization of mitochondria during P19 cell differentiation were investigated by electron micros- copy (Fig. 2). Clustering of mitochondria was observed in undifferentiated P19 cells, with individual mitochondria displaying granular and thread-like patterns. During the aggregation stage of differentiation, we observed that round mitochondria dispersed around the nucleus; these mitochondria appeared smaller compared to those of undifferentiated cells. Following this stage, the number of mitochondria increased, and they were found dispersed throughout the cytoplasm, with the majority appearing rod-shaped, with extended cristae.

Mitochondrial DNA copy number. The mtDNA copy number per mitochondrion is considered to be constant in all mammalian cell types (22). We examined the mtDNA copy number of the undifferentiated and differentiated P19 cells harvested at different time points using qPCR (Fig. 3). Our results show that the mtDNA copy number was significantly higher on days 5 and 7 (both $\mathrm{P}<0.05$ ) compared to earlier stages of differentiation, i.e. days 3 and 5, respectively, and 

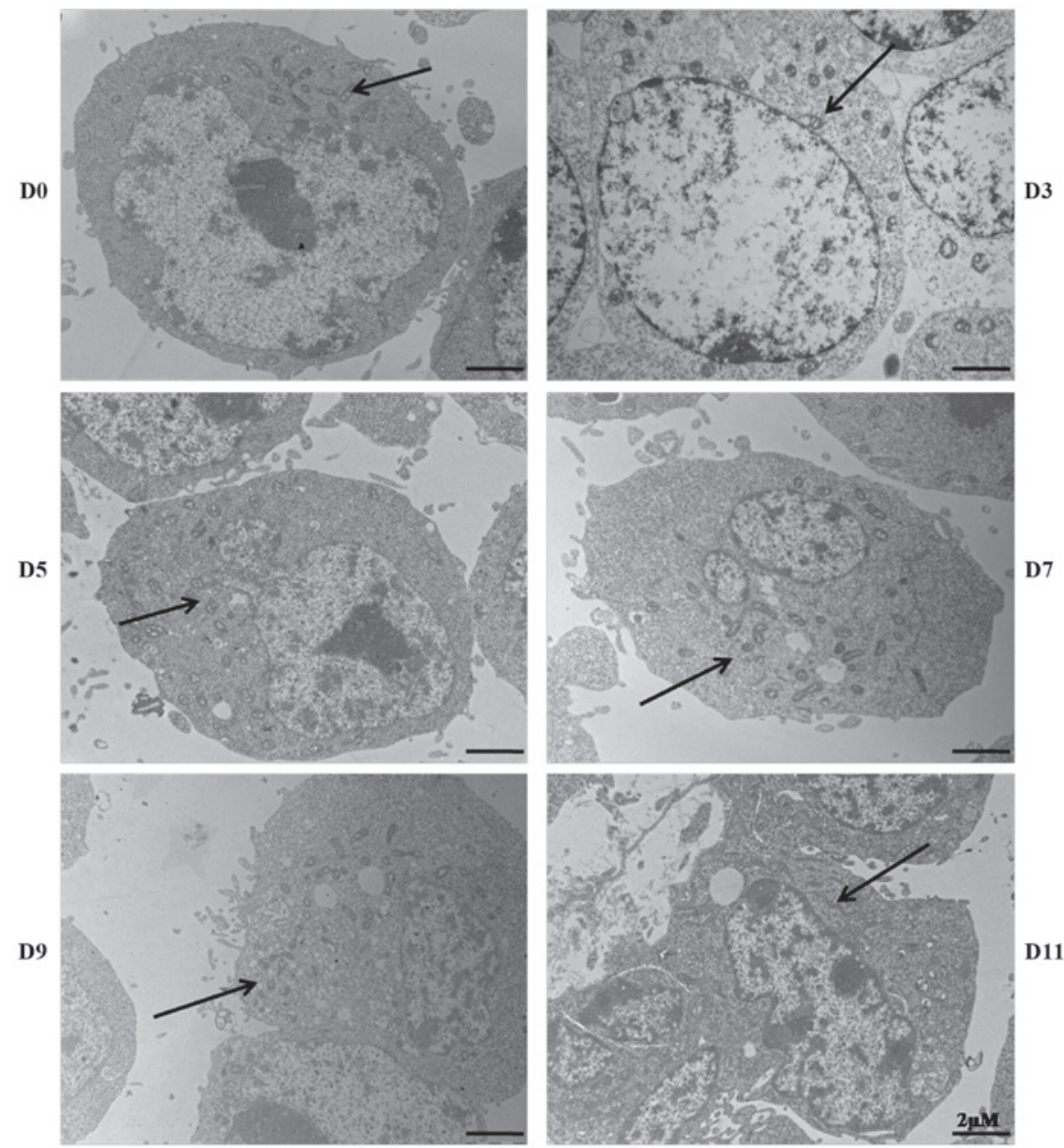

Figure 2. Mitochondrial morphology and localization in P19 cells throughout cardiomyocyte differentiation. Representative electron microscope images of mitochondria in differentiating P19 cells on day (D)0, D3, D5, D7, D9, and D11 (x10,000 magnification; scale bar, $2 \mu \mathrm{M}$ ).

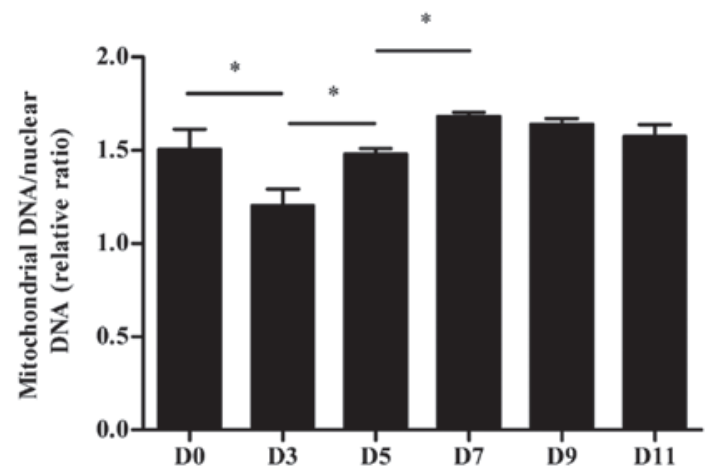

Figure 3. Cellular content in mitochondrial DNA (mtDNA) during differentiation of P19 cells into cardiomyocytes, on day (D)0, D3, D5, D7, D9, and D11. The mtDNA copy number was determined by quantitative PCR, with primers designed to target the cytochrome $\mathrm{B}(C Y T B)$ and $28 S$ rRNA genes. The ratio of $C Y T B$ to $28 s$ rRNA levels was used to estimate the concentration of mitochondria per cell. The results are presented as the mean \pm standard deviation of six experiments. ${ }^{*} \mathrm{P}<0.05$.

there was no significant difference in mtDNA copy number during the remaining stages of cardiomyocyte differentiation $(\mathrm{P}>0.05)$.

Intracellular ROS levels in differentiated P19 cells. The intracellular levels of ROS were measured in differentiating

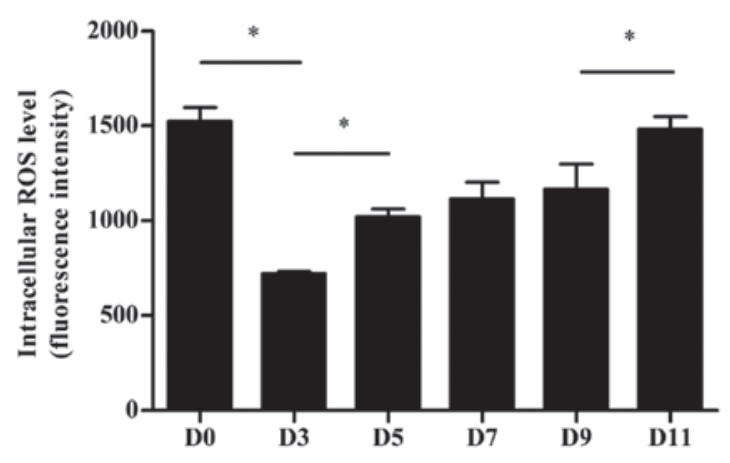

Figure 4. Intracellular concentration in reactive oxygen species (ROS) during differentiation of P19 cells into cardiomyocytes, on day (D)0, D3, D5, D7, D9, and D11. The ROS level was determined by fluorescence-activated cell sorting (FACS). The results are presented as the mean \pm standard deviation of six experiments. ${ }^{*} \mathrm{P}<0.05$.

P19 cells, using the fluorescent probe DCFDA (Fig. 4). Undifferentiated P19 cells had a relatively high intracellular ROS level, which was significantly reduced in aggregating cells on day $3(\mathrm{P}<0.05)$. From that day onwards, the ROS level gradually increased, and returned to baseline levels on day 11 . 


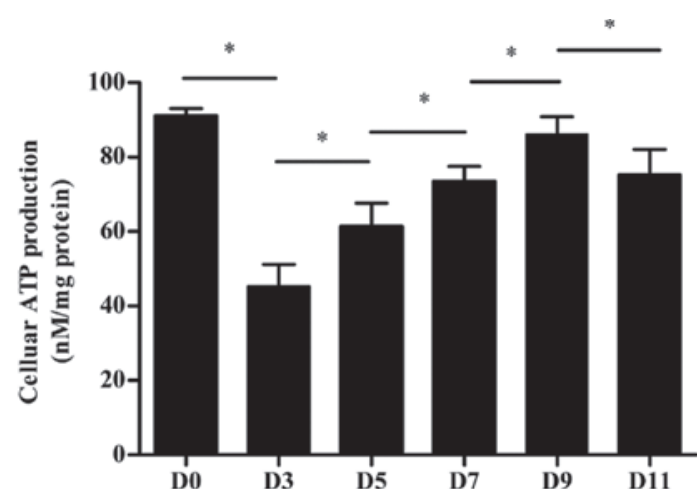

Figure 5. Intracellular adenosine triphosphate (ATP) production during differentiation of P19 cells into cardiomyocytes, on day (D)0, D3, D5, D7, D9, and D11. Cellular ATP production was normalized to the total protein concentration. The results are presented as the mean \pm standard deviation of six experiments. ${ }^{*} \mathrm{P}<0.05$.

Cellular ATP production. The concentration of cellular ATP was measured in differentiating P19 cells (Fig. 5). The intracellular ATP content of differentiated cells on day 3 was decreased compared to that of undifferentiated P19 cells on day $0(\mathrm{P}<0.05)$. On the following days, the intracellular ATP production gradually increased, although the total cellular ATP level was slightly decreased on day 11 compared to day 9 $(\mathrm{P}<0.05)$.

\section{Discussion}

Since adult human heart cells have very limited regenerative capacity, the application of stem cell-based cardiac regeneration to repair the failing heart has attracted a great deal of attention (23). ES cells, induced pluripotent stem cells, and embryonic carcinoma cells have become major models for the elucidation of the mechanisms underlying the process of differentiation into cardiomyocytes $(8,9)$. Due to the fact that the adult heart has a very high energy demand, as it continuously contracts to supply the body with blood (24), the role of mitochondria during cardiac differentiation has emerged as an important study area.

Advances in electron microscopy have allowed to reveal the considerable structural diversity of the mitochondria, which change shapes under different physiological conditions (25). These different configurations are believed to relate to mitochondrial function (26). In our study, mitochondria were clustered and appeared as granular and thread-like organelles in undifferentiated P19 cells, which is different from the mitochondrial morphology and distribution in ES cells (25). This difference in morphology may be explained by the origin of the P19 embryonal carcinoma cells. Given that they are derived from a teratocarcinoma induced in $\mathrm{C} 3 \mathrm{H} / \mathrm{HC}$ mice (10), these cells are expected to possess features of both tumor and stem cells. Accordingly, P19 have energy requirements consistent with the maintenance of the pluripotent state, as well as the need for continuous growth. Following the aggregation stage of differentiation, the dynamic distribution and morphology of the mitochondria were similar to that of mitochondria of human embryonic stem cells (hESCs) (25), and consistent with the energy demands of cells differentiating into cardiomyocytes.
Two key features of the mitochondria are the facts that they are the only organelles in animal cells containing their own genome, and that the multicopy mtDNA is maternally inherited (27). In this study, we observed an initial decline in the mtDNA copy number in aggregating cells relative to undifferentiated P19 cells, followed by a gradual increase back to baseline levels during differentiation. It is possible that the low mtDNA copy number in aggregating cells may be required to maintain their proliferative capacity in suspension culture, while the subsequent increase between days 3 and 7 is in accordance with the observed changes in mitochondrial distribution and morphology. We interpret the stable mtDNA copy number from day 7 onwards as an indication that the majority of P19 embryonal carcinoma cells had successfully committed to the cardiomyocyte lineage.

Mitochondria are considered to be the major cellular sources of ROS and ATP, and thus, the ROS and ATP cellular levels were here used as markers of mitochondrial function in the P19 cells differentiating into cardiomyocytes. Furthermore, the production of ROS by mitochondria (28) or the nonphagocytic NADPH oxidases (29), are known to play a key role in the differentiation of ES cells into the cardiomyocyte lineage (30-32). Consistent with the dynamic changes in mitochondrial number and mtDNA copy number, we observed that intracellular ROS dropped to its lowest level on day 3 of differentiation, and then gradually returned to baseline levels. As a metabolic by-product and a common signal in cellular processes, fluctuations in the intracellular ROS level may reflect changes in ROS signaling involved in P19 cell differentiation, or may be a consequence of the differentiation into high-energy demand cardiomyocytes. Similarly, intracellular ATP also declined to its lowest level on day 3, and then steadily increased as the process of differentiation into cardiomyocytes progressed and the energy demands became higher. Interestingly, on day 11 of differentiation, intracellular ATP production was again slightly, but significantly, reduced. We hypothesize that this late decrease in the ATP level reflects higher rates of ATP consumption relative to the rates of ATP synthesis, reflecting the increased energy demand in the beating cardiomyocyte.

In summary, our results demonstrate that during the differentiation of P19 embryonal carcinoma cells into cardiomyocytes, mitochondria undergo dynamic changes in their morphology and function. Specifically, we suggest that mitochondria of undifferentiated cells transform from a primitive status with immature morphology and low function into aggregating cells, and then cells with gradually enhanced function along the differentiation process. Our study is the first to indicate, to the best of our knowledge, that dynamic mitochondrial changes occur throughout the differentiation of P19 cell-derived cardiomyocytes. This study thus offers a glimpse into the mechanisms underlying P19 embryonal carcinoma cell differentiation into cardiomyocytes, and may be useful in the optimization of protocols for the in vitro differentiation of cardiomyocytes.

\section{Acknowledgements}

This study was supported by grants from the National Natural Science Foundation of China (no. 81070138), the 
Natural Science Foundation of Jiangsu Province, China (no. BK2010582), and the Talent Foundation of Jiangsu Province, China (no. WSN8020).

\section{References}

1. Gavin JB, Maxwell L and Edgar SG: Microvascular involvement in cardiac pathology. J Mol Cell Cardiol 30: 2531-2540, 1998.

2. Klingel K, Sauter M, Bock CT, Szalay G, Schnorr JJ and Kandolf R: Molecular pathology of inflammatory cardiomyopathy. Med Microbiol Immunol 193: 101-107, 2004.

3. Sabharwal NK and Lahiri A: Role of myocardial perfusion imaging for risk stratification in suspected or known coronary artery disease. Heart 89: 1291-1297, 2003.

4. Koudstaal S, Jansen Of Lorkeers SJ, Gaetani R, et al: Concise review: heart regeneration and the role of cardiac stem cells Stem Cells Transl Med 2: 434-443, 2013.

5. Itescu S, Schuster MD and Kocher AA: New directions in strategies using cell therapy for heart disease. J Mol Med (Berl) 81: 288-296, 2003.

6. Kehat I and Gepstein L: Human embryonic stem cells for myocardial regeneration. Heart Fail Rev 8: 229-236, 2003.

7. Lev S, Kehat I and Gepstein L: Differentiation pathways in human embryonic stem cell-derived cardiomyocytes. Ann NY Acad Sci 1047: 50-65, 2005.

8. van der Heyden MA and Defize LH: Twenty one years of P19 cells: what an embryonal carcinoma cell line taught us about cardiomyocyte differentiation. Cardiovasc Res 58: 292-302, 2003.

9. Wei H, Juhasz O, Li J, Tarasova YS and Boheler KR: Embryonic stem cells and cardiomyocyte differentiation: phenotypic and molecular analyses. J Cell Mol Med 9: 804-817, 2005.

10. McBurney MW, Jones-Villeneuve EM, Edwards MK and Anderson PJ: Control of muscle and neuronal differentiation in a cultured embryonal carcinoma cell line. Nature 299: 165-167, 1982.

11. Skerjanc IS: Cardiac and skeletal muscle development in P19 embryonal carcinoma cells. Trends Cardiovasc Med 9: 139-143, 1999.

12. Näveri L, Näveri $H$ and Härkönen $M$ : Myocardial energy metabolism. Ann Chir Gynaecol 76: 3-11, 1987.

13. Birky CW Jr: Uniparental inheritance of mitochondrial and chloroplast genes: mechanisms and evolution. Proc Natl Acad Sci USA 92: 11331-11338, 1995.

14. Anderson S, Bankier AT, Barrell BG, et al: Sequence and organization of the human mitochondrial genome. Nature 290 $457-465,1981$

15. Osellame LD, Blacker TS and Duchen MR: Cellular and molecular mechanisms of mitochondrial function. Best Pract Res Clin Endocrinol Metab 26: 711-723, 2012.

16. Bavister BD: The mitochondrial contribution to stem cell biology. Reprod Fertil Dev 18: 829-838, 2006.
17. Oh SK, Kim HS, Ahn HJ, et al: Derivation and characterization of new human embryonic stem cell lines: SNUhES1, SNUhES2, and SNUhES3. Stem Cells 23: 211-219, 2005.

18. St John JC, Ramalho-Santos J, Gray HL, et al: The expression of mitochondrial DNA transcription factors during early cardiomyocyte in vitro differentiation from human embryonic stem cells. Cloning Stem Cells 7: 141-153, 2005.

19. Edwards MK, Harris JF and McBurney MW: Induced muscle differentiation in an embryonal carcinoma cell line. Mol Cell Biol 3: 2280-2286, 1983.

20. Kaaman M, Sparks LM, van Harmelen V, Smith SR, Sjolin E, Dahlman I and Arner P: Strong association between mitochondrial DNA copy number and lipogenesis in human white adipose tissue. Diabetologia 50: 2526-2533, 2007.

21. Maxwell DP, Wang Y and McIntosh L: The alternative oxidase lowers mitochondrial reactive oxygen production in plant cells. Proc Natl Acad Sci USA 96: 8271-8276, 1999.

22. Robin ED and Wong R: Mitochondrial DNA molecules and virtual number of mitochondria per cell in mammalian cells. J Cell Physiol 136: 507-513, 1988.

23. Christoffels V: Regenerative medicine: muscle for a damaged heart. Nature 474: 585-586, 2011.

24. Poirier VL: Can we develop a permanent pulsatile rotary blood pump? Yes, we can. Artif Organs 20: 475-480, 1996.

25. Cho YM, Kwon S, Pak YK, et al: Dynamic changes in mitochondrial biogenesis and antioxidant enzymes during the spontaneous differentiation of human embryonic stem cells. Biochem Biophys Res Commun 348: 1472-1478, 2006.

26. Mannella CA: Structural diversity of mitochondria: functional implications. Ann NY Acad Sci 1147: 171-179, 2008.

27. Giles RE, Blanc H, Cann HM and Wallace DC: Maternal inheritance of human mitochondrial DNA. Proc Natl Acad Sci USA 77: 6715-6719, 1980

28. Crespo FL, Sobrado VR, Gomez L, Cervera AM and McCreath KJ: Mitochondrial reactive oxygen species mediate cardiomyocyte formation from embryonic stem cells in high glucose. Stem Cells 28: 1132-1142, 2010.

29. Buggisch M, Ateghang B, Ruhe C, Strobel C, Lange S, Wartenberg M and Sauer H: Stimulation of ES-cell-derived cardiomyogenesis and neonatal cardiac cell proliferation by reactive oxygen species and NADPH oxidase. J Cell Sci 120: 885-894, 2007.

30. Sauer H, Rahimi G, Hescheler J and Wartenberg M: Role of reactive oxygen species and phosphatidylinositol 3-kinase in cardiomyocyte differentiation of embryonic stem cells. FEBS Lett 476: 218-223, 2000.

31. Schmelter M, Ateghang B, Helmig S, Wartenberg M and Sauer H: Embryonic stem cells utilize reactive oxygen species as transducers of mechanical strain-induced cardiovascular differentiation. FASEB J 20: 1182-1184, 2006.

32. Sharifpanah F, Wartenberg M, Hannig M, Piper HM and Sauer H: Peroxisome proliferator-activated receptor alpha agonists enhance cardiomyogenesis of mouse ES cells by utilization of a reactive oxygen species-dependent mechanism. Stem Cells 26: 64-71, 2008 . 